\title{
References
}

Astbury, W. T. (1947). Cold Spring Harbour Symposium on Quantitative Biology, 12, 56.

British Association for the Advancement of ScIence (1937). Mathematical Tables, Vol. 6, Part 1. Cambridge: University Press.

Clapp, M. M. (1937). J. Math. Phys. 16, 76.

Cox, E. G. \& Shaw, W. F. B. (1930). Proc. Roy. Soc. A, 127,71 .

Franklin, R. E. \& Gosling, R. G. (1953a). Acta Cryst. 6,673 .

Acta Cryst. (1953). 6, 685

\section{The Fourier Transform of a Coiled-Coil}

\author{
By F. H. C. CRICK \\ The Medical Research Council Unit for the Study of the Molecular Structure of Bidological Systems, \\ The Cavendish Laboratory, Cambridge, England
}

(Received 14 March 1953)

The Fourier transforms are given for a continuous coiled-coil, and for a set of atoms spaced at regular intervals along a coiled-coil. The nature of the solution is briefly discussed.

\section{Introduction}

It has recently been suggested simultaneously by Pauling \& Corey (1953) and by Crick (1952) that the structure of $\alpha$-keratin may be based on a coiled-coil, i.e. on a helix with a small repeat whose axis has been slightly deformed so that it follows a larger more gradual helix. The small helix proposed is the $\alpha$-helix of Pauling, Corey \& Branson (195I).

It is therefore of interest to calculate the Fourier transform (or continuous structure factor) of structures of this sort. Those considered here are the continuous coiled-coil and the discontinuous coiled-coil. The former is an infinitely thin 'wire' of electron density, and the latter is a set of scattering points (atoms) placed at regular intervals on a coiled-coil locus. It will be shown that the two results are very closely related.

To obtain the structure factors for a structure of this type made up of real atoms, one follows a similar procedure to that described by Cochran, Crick \& Vand (1952) in calculating the transform of the simple $\alpha$-helix, i.e. one considers the atoms as being in sets, each set consisting of one atom from each residue. Thus all the nitrogen atoms of the polypeptide backbone will be in one set, all the oxygen atoms of the backbone in another, and so on. One then uses the formula derived in this paper to calculate the contribution of each set separately, allowance being made for the finite size of the atom by multiplying the result for a set of points by the appropriate atomic scattering factor in the usual way. The results are then added together, with proper allowance for phase, to give the structure factor for the complete structure.
The advantage of a general solution of the type given here is that instead of calculating the contribution of each atom separately one can group them into sets, in this case with a large number in each, and calculate the whole contribution of a set at one go.

We shall call the small helix the minor helix and the larger helix followed by its axis the major helix.

\section{Mathematical method}

A general description is given first, and the particular case of the coiled-coil is then derived afterwards.

Consider first the problem of a continuous infinitely thin 'wire' of electron density. Let us suppose that it is defined parametrically in terms of a parameter, $t$, which may be proportional to the length along the wire, though this is not essential. We also assume that the structure repeats exactly after a distance $c$ in the $z$ direction.

We can form the expression for the value of the Fourier transform of such a wire at some particular point in reciprocal space. We will call this $C(R, \psi, Z)$, where $R, \psi$ and $Z$ are the cylindrical co-ordinates of the point in reciprocal space under consideration.

Now it will often happen that the expression for the transform at this point will be an integral of the form

$$
C(R, \psi, Z)=\int_{0}^{t_{0}} f_{1}(t) f_{2}(t) f_{3}(t) d t
$$

where $t_{0}$ is the value of $t$ after which the structure repeats, and $f_{1}(t), f_{2}(t)$ and $f_{3}(t)$ are simple functions of $t$. They may also be functions of $R, \psi$ and $Z$, but for the moment we are considering these as fixed. 
We can evaluate this integral by Fourier methods. To do this we consider the Fourier transform of these functions, considered as functions of $t$, i.e. we define

$$
F(T) \equiv \frac{1}{t_{0}} \int_{0}^{t_{0}} f(t) \exp (2 \pi i t T) d t
$$

where $T$ is the co-ordinate in reciprocal parametric space. If $f(t)$ repeats itself exactly $m$ times in the interval $t_{0}$ then we need only consider $T$ at integer multiples of $m / t_{0}$.

We can now obtain the integral we require by the repeated use of Parseval's theorem.

The $p$ th component of the transform of the product $f_{1}(t) \cdot f_{2}(t)$-its value at $p T_{0}$-is given by

$$
\sum_{n} F_{1}\left(n T_{0}\right) F_{2}\left(\overline{p-n} \cdot T_{0}\right)
$$

where $T_{0}=1 / t_{0}$ and the sum is taken over all integer values of $n$.

We also have the relation

$$
\int_{0}^{t_{0}} f_{1}(t) f_{2}(t) d t=t_{0} \sum_{n} F_{1}\left(n T_{0}\right) F_{2}\left(-n T_{0}\right) .
$$

By repeated application of these results we arrive at

$$
\begin{aligned}
& \int_{0}^{t_{0}} f_{1}(t) f_{2}(t) f_{3}(t) d t \\
& \quad=t_{0} \sum_{p} \sum_{q} F_{1}\left(q T_{0}\right) F_{2}\left(\overline{p-q} \cdot T_{0}\right) F_{3}\left(-p T_{0}\right) .
\end{aligned}
$$

This can be written in the alternative more symmetrical form

$$
t_{0} \sum_{p} \sum_{q} \sum_{s} F_{1}\left(p T_{0}\right) F_{2}\left(q T_{0}\right) F_{3}\left(s T_{0}\right),
$$

subject to the restriction that we only consider terms for which

$$
p+q+s=0 .
$$

If we now use the fact that $f_{1}(t)$ repeats $m_{1}$ times in $t_{0}, f_{2}(t) m_{2}$ times, etc. we obtain

$$
\begin{aligned}
& C(R, \psi, Z) \\
& \quad=\sum_{p} \sum_{q} \sum_{s} F_{1}\left(m_{1} p T_{0}\right) F_{2}\left(m_{2} q T_{0}\right) F_{3}\left(m_{3} s T_{0}\right),
\end{aligned}
$$

with the restriction

$$
m_{1} p+m_{2} q+m_{3} s=0 .
$$

The summations go over all integer values of $p, q$ and $s$, both positive and negative, satisfied by equation (3).

If instead of a continuous wire we have a set of points located at regular intervals along the path of the wire, one point being at $t=0$, we obtain, instead of an integral, a sum of the form

$$
\sum_{n=0}^{M-1} f_{1}\left(n t^{\prime}\right) f_{2}\left(n t^{\prime}\right) f_{3}\left(n t^{\prime}\right) \text {. }
$$

We have assumed that each point occurs after an interval $t^{\prime}$, and that there are $M$ points in the interval $t_{0}$, so that $M t^{\prime}=t_{0}$.
The discontinuous function can be obtained from the continuous one by multiplying it by the appropriate delta function. The transform is obtained from the corresponding folding process in reciprocal parametric space. The solution then follows very similar lines to those already outlined.

It turns out that one arrives at the same equation for the Fourier transform as before, namely equation (2), but that the restriction now takes the form

$$
m_{1} p+m_{2} q+m_{3} s=m^{\prime} M,
$$

where $m^{\prime}$ can take any integer value, positive or negative. Thus the continuous case may be considered as a special case of the discontinuous case, obtainable by putting $m^{\prime}=0$.

The very symmetrical form of our answer shows that we are not restricted to three functions; we can easily add further functions, and the new answer can be written down from inspection. Thus we have a very general solution for any structure which can be expressed in parametric form. For a non-uniform wire we can always include an expression proportional to the weight of the wire at that point.

\section{Parametric equation of a continuous coiled-coil}

Let the major helix-i.e. the one with the larger repeat-be a right-handed helix defined by

$$
\left.\begin{array}{l}
x=r_{0} \cos \omega_{0} t, \\
y=r_{0} \sin \omega_{0} t, \\
z=P\left(\omega_{0} t / 2 \pi\right),
\end{array}\right\}
$$

where $\omega_{0}$ is positive.

This is a helix of radius $r_{0}$ and a repeat distance of $P$ in the $z$ direction. The pitch angle, $\alpha$, is given by $\tan \alpha=2 \pi r_{0} / P$.

Now imagine a new set of orthogonal axes $x^{\prime}, y^{\prime}, z^{\prime}$ defined for a given value of $t$ as follows:

(a) The origin of the new frame is at the point $(a, b, c)$ in the old frame, and satisfies equation (5).

(b) The $z^{\prime}$ axis is tangential to the major helix.

(c) The $x^{\prime}$ axis lies in a plane perpendicular to the $z$ axis.

Thus as $t$ varies these new axes follow the major helix, the $x^{\prime}$ axis always pointing directly away from the fibre axis, $z$. Consider a point $\left(x^{\prime}, y^{\prime}, z^{\prime}\right)$ in the new frame. What are its co-ordinates in the old frame?

$$
\begin{aligned}
& \text { We obtain } \\
& x-a=x^{\prime} \cos \omega_{0} t-y^{\prime} \cos \alpha \cdot \sin \omega_{0} t-z^{\prime} \sin \alpha \cdot \sin \omega_{0} t, \\
& y-b=x^{\prime} \sin \omega_{0} t+y^{\prime} \cos \alpha \cdot \cos \omega_{0} t+z^{\prime} \sin \alpha \cdot \cos \omega_{0} t, \\
& z-c=\quad-y^{\prime} \sin \alpha \quad+z^{\prime} \cos \alpha .
\end{aligned}
$$

Let us now make the point in the new frame rotate rapidly with $t$ to trace out the minor helix, so that

$$
\begin{aligned}
& x^{\prime}=r_{1} \cos \omega_{1} t, \\
& y^{\prime}=r_{1} \sin \omega_{1} t, \\
& z^{\prime}=0 .
\end{aligned}
$$


If $\omega_{1}$ is positive the minor helix is right-handed. If negative, left-handed.

Thus we finally get for the equation of a coiled-coil in the original frame

$$
\begin{aligned}
& x= \\
& r_{0} \cos \omega_{0} t+r_{1} \cos \omega_{0} t \cdot \cos \omega_{1} t-r_{1} \cos \alpha \cdot \sin \omega_{0} t \cdot \sin \omega_{1} t \\
& y= \\
& r_{0} \sin \omega_{0} t+r_{1} \sin \omega_{0} t \cdot \cos \omega_{1} t+r_{1} \cos \alpha \cdot \cos \omega_{0} t \cdot \sin \omega_{1} t \\
& \quad z=P\left(\omega_{0} t / 2 \pi\right)-r_{1} \sin \alpha \cdot \sin \omega_{1} t \cdot
\end{aligned}
$$

Put

so that

$$
\bar{r}_{1}+\Delta=r_{1}, \bar{r}_{1}-\Delta=r_{1} \cos \alpha
$$

$$
\bar{r}_{1}=r \cdot(1+\cos \alpha) / 2, \Delta=r_{1}(1-\cos \alpha) / 2,
$$

and we easily obtain

$$
\begin{aligned}
& x=r_{0} \cos \omega_{0} t+\bar{r}_{1} \cos \left[\left(\omega_{0}+\omega_{1}\right) t\right]+\Delta \cos \left[\left(\omega_{0}-\omega_{1}\right) t\right] \\
& y=r_{0} \sin \omega_{0} t+\bar{r}_{1} \sin \left[\left(\omega_{0}+\omega_{1}\right) t\right]+\Delta \sin \left[\left(\omega_{0}-\omega_{1}\right) t\right], \\
& z=P\left(\omega_{0} t / 2 \pi\right)-r_{1} \sin \alpha \cdot \sin \omega_{1} t .
\end{aligned}
$$

We now neglect $\Delta$, since for cases we are likely to consider it is very small. The theory can easily be extended to include it, and this has been done in the full result given later in equation (13).

We shall now assume that while the major helix makes exactly $N_{0}$ turns in the repeat distance $c$, the minor helix makes exactly $N_{1}$ turns in its own coordinate frame. We shall also restrict ourselves to the case of a left-handed minor helix and a right-handed major helix, so that $-\omega_{1} / \omega_{0}=N_{1} / N_{0}$. Thus our parametric equation for the coiled-coil becomes

$$
\left.\begin{array}{l}
x=r_{0} \cos \omega_{0} t+\bar{r}_{1} \cos \left[\left(N_{1} / N_{0}-1\right) \omega_{0} t\right], \\
y=r_{0} \sin \omega_{0} t-\bar{r}_{1} \sin \left[\left(N_{1} / N_{0}-1\right) \omega_{0} t\right], \\
z=P\left(\omega_{0} t / 2 \pi\right)+r_{1} \sin \alpha \sin \left[\left(N_{1} / N_{0}\right) \omega_{0} t\right] .
\end{array}\right\}
$$

The structure repeats after a distance $N_{0} P$ in the $Z$ direction. We have $c=N_{0} P$.

\section{The transform of the continuous coiled-coil}

We write, as usual, for the transform at the point $(X, Y, Z)$ in reciprocal space:

$$
C(X, Y, Z)=\int_{0}^{t_{0}} \exp (2 \pi i[x X+y Y+z Z]) d t,
$$

since we need only integrate over the wire because the electron density elsewhere is zero. We now substitute from (6) into (7), and putting

$$
R^{2}=X^{2}+Y^{2}, \tan \psi=Y / X,
$$

we easily obtain

$$
\begin{gathered}
C(R, \psi, Z)=\int_{0}^{t_{0}} \exp 2 \pi i\left\{R r_{0} \cos \left(\omega_{0} t-\psi\right)\right. \\
+R \bar{r}_{1} \cos \left[\left(N_{1} / N_{0}-1\right) \omega_{0} t+\psi\right] \\
\left.+Z r_{1} \sin \alpha \cos \left[\left(N_{1} / N_{0}\right) \omega_{0} t-\pi / 2\right]+Z P\left(\omega_{0} t / 2 \pi\right)\right\} d t
\end{gathered}
$$

Notice that since the structure repeats after a distance $c$ in the $Z$ direction, the transform will be non-zero only when $Z=l / c$, where $l$ is an integer.

Equation (8) is in the general form we have discussed earlier, namely

$$
C(R, \psi, Z)=\int_{0}^{t_{0}} f_{1}(t) f_{2}(t) f_{3}(t) f_{4}(t) d t
$$

where we define

$$
\begin{aligned}
& f_{1}(t) \equiv \exp \left[2 \pi i R r_{0} \cos \left(\omega_{0} t-\psi\right)\right], \\
& f_{2}(t) \equiv \exp \left[2 \pi i R \bar{r}_{1} \cos \left\{\left(N_{1} / N_{0}-1\right) \omega_{0} t+\psi\right\}\right], \\
& f_{3}(t) \equiv \exp \left[2 \pi i Z r_{1} \sin \alpha \cdot \cos \left\{\left(N_{1} / N_{0}\right) \omega_{0} t-\pi / 2\right\}\right], \\
& f_{4}(t) \equiv \exp \left[2 \pi i Z P\left(\omega_{0} t / 2 \pi\right)\right] .
\end{aligned}
$$

We obtain the corresponding transform of the first three of these by using the identity

$$
\int_{0}^{2 \pi} \exp (i w \cos \theta) \exp (i n \theta) d \theta=2 \pi i^{n} J_{n}(w)
$$

for integral $n$, where $J_{n}(w)$ is the Bessel function of order $n$.

If $f(t)$ can be written in the form

$$
f(t)=\exp [i w \cos (\alpha t+\beta)],
$$

and if it repeats $m$ times in the interval $t_{0}$, then from (1) and (9) we eventually obtain

$$
F\left(n . m / t_{0}\right)=J_{n}(w) \exp (-i n \beta+i n \pi / 2),
$$

where $n$ is an integer. Note that $\alpha t_{0}=2 \pi m$.

This case covers $f_{1}, f_{2}$ and $f_{3}$. To obtain $F_{4}(T)$ we write

$$
F_{4}(T)=\frac{1}{t_{0}} \int_{0}^{t_{0}} \exp \left(2 \pi i Z P\left(\omega_{0} t / 2 \pi\right)\right) \exp (2 \pi i t T) d t .
$$

This is zero except when the two exponentials cancel, i.e. when $Z P\left(\omega_{0} / 2 \pi\right)=-T$. Now $N_{0} P=c$, the repeat distance of the structure. If we write $Z=l / c$ (so that $l$ is the number of the layer-line), we obtain

$$
F_{4}(T)=1 \text { when } T=-l / t_{0} .
$$

Applying these results, and using the general formula of equations (2) and (3), we finally obtain

$$
\begin{aligned}
C(R, \psi, l / c)= & \sum_{p} \sum_{q} \sum_{s} J_{p}\left(2 \pi R r_{0}\right) J_{q}\left(2 \pi R \bar{r}_{1}\right) J_{s}\left(2 \pi(l / c) r_{1} \sin \alpha\right) \\
& \times \exp i[p(\pi / 2+\psi)+q(\pi / 2-\psi)+s \pi], \quad(10)
\end{aligned}
$$

the sums to be taken over all integer values for which

$$
N_{0} p+\left(N_{1}-N_{0}\right) q+N_{1} s=l .
$$

This has been normalized to make $F_{000}$ unity. The solution is of course only non-zero on a set of planes corresponding to integer values of $l$, since the structure is periodic in the $z$ direction but non-periodic in the other directions.

\section{The discontinuous coiled-coil}

This is the case of a set of points placed at regular intervals on a coiled-coil, the scattering being due to 
the points alone. We assume that one atom is at the point defined by $t=0$, and that there are $M$ atoms, spaced at regular intervals of $t$, in the complete repeat distance, $c$.

As already explained, the formula is identical with (10), except that instead of (11) we now have the restriction

$$
N_{0} p+\left(N_{1}-N_{0}\right) q+N_{1} s=l+M m^{\prime},
$$

where $m^{\prime}$ can take any integer value. The continuous case is thus the discontinuous case with $m^{\prime}$ restricted to zero.

The above results apply to a right-handed major helix and a left-handed minor helix. The result for both helices being right-handed can be obtained simply by giving $N_{1}$ a negative value. The identity

$$
J_{-n}(w)=J_{n}(w) \exp (i n \pi)
$$

is useful in recasting the formula.

The formula quoted assumes a rather special choice of the phase of both the major and minor helices. The general solution given at the end of this paper differs only in the exponential phase term, apart from the fact that we have assumed here that $\Lambda$ is negligible.

\section{The nature of the solution}

The solution looks more complicated than it is. This is because, as explained in the case of the simple helix (Cochran, Crick \& Vand, 1952), it is a property of Bessel functions that $J_{n}(x)$ is vanishingly small for small $x$ if $n$ is large. Thus for the central regions of reciprocal space, where $R$ is not too big, and for structures of a limited size, so that $r$ is also limited, $(2 \pi R r)$ is often fairly small. Thus $J_{n}(2 \pi R r)$ vanishes for high values of $n$, and we usually only have to consider the loworder Bessel functions.

The solution, then, says that the structure factor at the point in reciprocal space under consideration is given by the sum of an infinite numbers of terms, each of which is the product of three Bessel functions, multiplied by a phase factor. However, if one of the Bessel functions in any of the triple products is vanishingly small, that product also vanishes, so that the great majority of the triple products are effectively zero, and one usually only has to consider a very small number of them.

The solutions quoted are for a single continuous coiled-coil, or a single discontinuous set of atoms on a coiled-coil. As explained in the introduction, to obtain the structure factor for a number of sets of atoms one must calculate the structure factor for each set, and then add the structure factors together with due regard for phase.

If we examine the general solution in the light of the solution for a simple helix (Cochran, Crick \& Vand, 1952) we see that in a loose way we may think of the first Bessel term as due to the major helix, the second to the minor helix, and the third to the tilt produced on the minor helix by the major helix. Some of the important terms correspond to the rather obvious approximations that one would make if one did not have the full theory, and it is one of the advantages of the full theory that it enables one to see how far such approximations are justified.

In order to bring out the nature of the solution, it is best to discuss a simple case in outline. This is done in a separate paper (Crick, 1953). For the calculation of structure factors the full formula is required. This is given in the next section.

\section{The calculation of structure factors}

For this one requires the full solution. For a major helix given by

$$
\begin{aligned}
& x=r_{0} \cos \left(\omega_{0} t+\varphi_{0}\right), \\
& y=r_{0} \sin \left(\omega_{0} t+\varphi_{0}\right), \\
& z=P\left(\omega_{0} t / 2 \pi\right)+z_{0},
\end{aligned}
$$

and a minor helix given in its own rotating frame of reference by

$$
\begin{aligned}
& x^{\prime}=r_{1} \cos \left(\omega_{1} t+\varphi_{1}\right), \\
& y^{\prime}=r_{1} \sin \left(\omega_{1} t+\varphi_{1}\right), \\
& z^{\prime}=0,
\end{aligned}
$$

and with $M$ atoms in the repeat distance $c$, one atom being at the point $t=t_{1}$, we can show that if we put $\varphi_{M}=2 \pi M t_{1} / t_{0}$ then the Fourier transform for this set of $M$ atoms, normalized to make $F_{000}$ unity, is

$$
\begin{aligned}
& C(R, \psi, l / c) \\
= & \sum_{p} \sum_{q} \sum_{s} \sum_{d} J_{p}\left(2 \pi R r_{0}\right) J_{q}\left(2 \pi R \bar{r}_{1}\right) J_{s}\left(2 \pi(l / c) r_{1} \sin \alpha\right) \\
\times & J_{d}(2 \pi R \Delta) \exp \left[i p\left(\psi-\varphi_{0}+\pi / 2\right)+i q\left(-\psi+\varphi_{1}+\pi / 2\right)\right. \\
+ & \left.i s\left(-\varphi_{1}+\pi\right)+i d\left(\psi+\varphi_{1}+\pi / 2\right)-i m^{\prime} \varphi_{M}+2 \pi i l z_{0} / c\right],
\end{aligned}
$$

subject to the condition that

$$
N_{0} p+\left(N_{1}-N_{0}\right) q+N_{1} s+\left(N_{0}+N_{1}\right) d=l+M m^{\prime} .
$$

Here, as before, $N_{0}$ is the number of turns of the righthanded major helix in the repeat distance $c$, and $N_{1}$ is the number of turns of the left-handed minor helix in its own frame of reference in the same distance. Thus $-\omega_{1} / \omega_{0}=N_{1} / N_{0}$. The parameters $\Delta$ and $\bar{r}_{1}$, are defined on page 687, and the pitch angle $\alpha$ on page 686.

The transform of the corresponding continuous helix is obtained by taking $m^{\prime}=\varphi_{M}=0$.

It may happen that we have available the atomic co-ordinates of the basic straight left-handed helix from which the coiled-coil is formed. If the coiled-coil deformation is small, we can use these co-ordinates to obtain the values of the parameters we require with sufficient accuracy for most purposes.

Let us call the co-ordinates of the particular atom under consideration $\left(r_{s}, \varphi_{s}, z_{s}\right)$, taking $z_{s}$ as small as possible. We shall imagine the frame of reference to which these co-ordinates refer to be moved in space 
until it coincides with the position of our $\left(x^{\prime}, y^{\prime}, z^{\prime}\right)$ frame at $t=0$, i.e. with its origin at $\left(r_{0}, \varphi_{0}, z_{0}\right)$ in our basic frame and with its $x$ axis perpendicular to our basic $z$ axis and pointing directly away from it. It can be shown that we should put

$$
\begin{aligned}
& r_{1}=r_{s}, \\
& \varphi_{M}=2 \pi M\left(z_{s} / c\right) \cos \alpha, \\
& \varphi_{1}=\varphi_{s}+\left\{\left(N_{1}-N_{0}\right) / M\right\} \varphi_{M} .
\end{aligned}
$$

It should be remembered that $\left(r_{s}, \varphi_{s}, z_{s}\right)$ should refer to a left-handed helix in a right-handed frame.
I should like to thank Mr G. Kreisel for a number of interesting discussions and in particular for suggesting the use of Parseval's theorem at a crucial point.

\section{References}

Cochran, ${ }^{r}$., CRICK, F. H. C. \& VAND, V. (1952). Acta Cryst. 5, 581.

Crick, F. H. C. (1952). Nature, Lond. 170, 882.

CrICK, F. H. C. (1953). Acta Cryst. 6, 689.

Pauling, L. \& Corey, R. B. (1953). Nature, Lond. 171, 59.

Pauling, L., Corey, R. B. \& Branson, H. R. (1951). Proc. Nat. Acad. Sci., Wash. 37, 205.

Acta Cryst. (1953). 6, 689

\title{
The Packing of $\alpha$-Helices: Simple Coiled-Goils
}

\author{
By F. H. C. CRICK \\ The Medical Research Council Unit for the Study of the Molecular Structure of Biological \$systems, \\ The Cavendish Laboratory, Cambridge, England
}

(Received 14 March 1953)

\begin{abstract}
It is shown that when $\alpha$-helices of the same sense pack together they will probably do so about $20^{\circ}$ away from parallel. For very long chains this may lead to a coiled-coil. The two simplest models - the two-strand rope and the three-strand rope--are described, and used to illustrate the diffraction theory already developed. It is shown that they would give a diffuse $\alpha$-pattern. Possible examples of these models are briefly discussed.
\end{abstract}

\section{'Introduction}

It is now firmly established that the structure of the synthetic polypeptide polymethylglutamate is based on the $\alpha$-helix of Pauling, Corey \& Branson (1951). The general similarity of the $\mathrm{X}$-ray diffraction pattern of all synthetic polypeptides in the $\alpha$ form so far examined makes it very probable that they are all based on this helix.

Pauling \& Corey (1951) suggested that the $\alpha$-helix could also explain the ' $\alpha$-keratin' diffraction pattern, which the pioneer work of Astbury and his school has shown to be given in various degrees of perfection by porcupine quill, hair, horn, muscle, epidermin, fibrinogen and related proteins. (The $\mathrm{k}-\mathrm{m}-\mathrm{e}-\mathrm{f}$ group).

The main characteristics of this diffraction pattern are meridional arcs at spacings of about 5.15 and $1.5 \AA$, and a group of reflexions on and near the equator at spacings around $10 \AA$ (McArthur, 1943; Perutz, 1951). The two main difficulties in fitting the $\alpha$-helix to this structure are:

(1) The 5.15 $\AA$ reflexion on or very close to the meridian of the reciprocal lattice. A straight perfect $\alpha$-helix, parallel to the fibre axis, gives a strong layerline at a spacing of $5 \cdot 4 \AA$, but the intensity on the actual axis of the reciprocal lattice should be zero. This argument is less precise if the arrangement of the side-chains is not strictly helical.
(2) The density. If the centre of the broad equatorial reflexion at $9.8 \AA$ is taken as the (10.0) reflexion of a simple hexagonal lattice the calculated density for $\alpha$-keratin is too low. (It is not clear that all members of Astbury's $\mathrm{k}-\mathrm{m}-\mathrm{e}-\mathrm{f}$ group have a high density.)

To explain the 5.15 reflexion on the meridian Crick (1952) suggested that the $\alpha$-helix might be deformed into a coiled-coil. It was shown that the energy involved in this deformation was likely to be small. The reason suggested for the deformation was that the non-integer nature of the $\alpha$-helix made it more likely that two helices having the same sense of twist would pack together at an angle rather than exactly parallel, and that this would lead to a coiled-coil.

Simultaneously Pauling \& Corey (1953) put forward a detailed model for $\alpha$-keratin based on coiled-coils. They suggested that the origin of the deformation was a repeating sequence of animo acids, a repeat every seventh residue being required for two-thirds of the $\alpha$-helices in the structure, and a repeat every fourth residue for two-ninths of them.

This model can explain the simultaneous existence of both the $5 \cdot 15$ and the $1.5 \AA$ reflexions on the meridian. It also broadly explains the equatorial reflexions, and probably the near equatorial reflexions of African porcupine quill. The density calculated for this model is nearer the observed value, but it is still on the low side. 